\title{
Ultrasound-guided erector spinae plane block for acute pain management in patients undergoing posterior lumbar interbody fusion under general anaesthesia
}

\author{
MR El Ghamry, ${ }^{1}$ AS Elgebaly, ${ }^{1}$ AG Anwar, ${ }^{1}$ MN Shaddad ${ }^{2}$ \\ ${ }^{1}$ Anesthesia, Surgical Intensive Care and Pain Medicine, Faculty of Medicine, Tanta University, Egypt \\ 2 Department of Neurosurgery, Faculty of Medicine, Tanta University, Egypt \\ Corresponding author, email: drmonagh19802000@gmail.com
}

Background: The article dealt with evaluating the efficacy of bilateral single shot ultrasound-guided (US-guided) lumbar erector spinae plane block (ESPB) in patients scheduled for L3-L5 posterior lumbar interbody fusion (PLIF) under general anaesthesia (GA). The primary goal of the article was to determine total morphine consumption 24 hours postoperative. Secondary goals included determining total intraoperative and postoperative opioid consumption, length of post-anaesthesia care unit (PACU) stay, and complications.

Methods: Sixty patients, American Society of Anesthesiologists (ASA) I and II, aged 18-60 years, undergoing PLIF under GA were enrolled in this prospective, randomised, double-blinded study. Patients were randomised to 2 groups (30 patients each). Group I (control group) received GA only and group II received preoperative bilateral ESPB with $20 \mathrm{ml} 0.25 \%$ bupivacaine. The primary outcome was postoperative morphine consumption. Secondary measurements were intraoperative fentanyl consumption, time to first analgesic request, static and dynamic visual analogue score (VAS), haemodynamic changes, PACU stay, and complications. Results: Patients who received ESPB showed a significant decrease in intraoperative and postoperative opioid consumption, shortened PACU stay, and haemodynamic stability compared to those who received GA only. Significant increase of VAS was observed in group I compared with group II up to 8 and 12 hours postoperative (static and dynamic VAS respectively).

Conclusion: Preoperative bilateral single shot US-guided ESPB provided safe and effective postoperative analgesia for PLIF with reduced opioid consumption and short PACU stay.

Keywords: Erector spinae plane block, bupivacaine, ultrasound, lumbar interbody fusion, spondylolisthesis

\section{Introduction}

Patients complaining of spondylolisthesis often experience severe pain which reduces their quality of life. Failure to achieve adequate pain relief with conservative management therapy makes surgical intervention necessary and posterior lumbar interbody fusion (PLIF) is the management of choice. ${ }^{1}$

Control of postoperative spine surgery pain remains a challenge for the anaesthesiologist. In addition to incisional pain, these patients experience pain arising from deeper tissues, such as bones, ligaments, muscles, intervertebral disks, facet joints and damaged nerve roots. The pain from these structures may be severe and can lead to neural sensitisation and release of mediators both peripherally and centrally. The problem is that many of these patients are either opioid dependent or opioid tolerant, making them less responsive to the most commonly used therapy for postoperative pain (opioid-based intermittent or patient-controlled analgesia). ${ }^{2}$

There are many studies describing different modalities of acute postoperative pain relief which range from acute pain relief procedures, such as neuraxial analgesia, paravertebral block (PVB) and local infiltration for different nerves as supplementary analgesia with general anaesthesia, to systemically administered analgesia., ${ }^{3,4}$ In the current era of fast-tracking in spine surgery, optimal perioperative pain management plays a vital role. ${ }^{5}$
One of the first examples of erector spinae plane block (ESPB) was presented for thoracic analgesia in both chronic neuropathic pain as well as acute postsurgical or posttraumatic pain. Recently, considerable attention has been paid to ESPB, as it is a simple and safe technique thanks to easily identifiable ultrasonographic landmarks as well as low risk for serious complications, because the injection is into a tissue plane that is distant from pleura, major blood vessels and discrete nerves. ${ }^{6}$

Recent reports stated that ESPB is effective during surgical interventions and strengthens perioperative analgesia when done at lower thoracic vertebrae. ${ }^{1}$ To our knowledge, few publications are available which address the use of ESPB in spine surgeries. The aim of this study is to evaluate the efficacy of bilateral single shot ultrasound-guided ESPB for acute postoperative pain management in patients with double level lumbar spondylolisthesis (L3-L5) and candidates for posterior lumbar interbody fusion (PLIF) surgery under general anaesthesia.

\section{Materials and methods}

This prospective, double-blinded, randomised, controlled study was done from January 2018 to January 2019 in Tanta University Hospital after approval from our institutional ethical committee and obtaining the patients' written informed consent. Sixty male and female patients were enrolled in this study, whose age ranged from 18-60 years, with American Society of 
Anesthesiologists (ASA) physical status I or II. Patients were complaining from double level lumbar spondylolisthesis (L3L5) and scheduled for elective PLIF under general anaesthesia. The trial followed the CONSORT 2010 statement guidelines for conducting a randomised controlled trial. (Figure 1)

Exclusion criteria included patient's refusal, BMl $>35 \mathrm{~kg} / \mathrm{m}^{2}$, infection at the site of the needle puncture, allergy to any of the study drugs, coagulopathy, and uncooperative patients or who cannot express pain via visual analogue scale (VAS). Patients were randomised into two equal groups (30 patients in each) using computer-generated random numbers concealed in picking up sealed envelopes indicating the group of the assignment. Group I (control/no block) received only GA, group II (ESPB) received bilateral single shot US-guided ESP block with $20 \mathrm{ml} 0.25 \%$ bupivacaine.

The drug was prepared by an anaesthesiologist who was not involved in data collection, while another anaesthesiologist who conducted general anaesthesia and collected the study data was blind to the type of the solution injected in regional block until the end of the study. The patients were also blind to the type of injected solution.

Before enrolment to the study, a pre-anaesthetic visit was done for history taking, physical examination, and routine laboratory investigations. Instructions about the use of the $100 \mathrm{~mm}$ visual analogue scale (VAS) for pain assessment and bilateral single shot ESPB technique were fully explained.

On arrival at the operating room (OR), 18-gauge intravenous peripheral cannula was inserted, and monitoring (Cardiocaps/5; DatexOhmeda, Helsinki, Finland) was applied, including electrocardiogram (ECG), non-invasive blood pressure (NIBP) and pulse oximetry; then the patients were premedicated using a sedative dose of midazolam $0.05 \mathrm{mg} / \mathrm{kg}$ to facilitate ESPB, dexamethasone $8 \mathrm{mg}$ IV and ondansetron $4 \mathrm{mg}$ IV (prophylactic anti-emetic).

After recording baseline heart rate (HR), mean blood pressure (MBP) and oxygen saturation $\left(\mathrm{SpO}_{2}\right)$, patients were placed in the left lateral position and the block applied to the upper side. Two to five $\mathrm{MHz}$ ultrasound (US) probe (Phillips cx50 extreme edition; USA) was used for all blocks, which were done by the same anaesthesiologist who is an expert in the US-guided nerve block. The probe was covered with a sterile cover. Transverse process of vertebrae and erector spinae muscle were identified with the US probe placed in paramedian sagittal plane, $3 \mathrm{~cm}$ lateral to the spinous process of 3rd lumbar vertebrae (L3).

\section{ESPB technique}

Under aseptic technique and after infiltration with $5 \mathrm{ml}$ of $1 \%$ lidocaine, a 22G, $50 \mathrm{~mm}$, insulated facet type needle (visioplex ${ }^{\circledast}$ - vygon - France) was introduced in an in-plane approach, a cephalic to caudal direction until L3 transverse process was hit, then the needle was slightly withdrawn. Confirmation of the correct position of the needle tip was done by injecting 0.5-1 $\mathrm{ml}$ of local anaesthetic (LA). Correct needle tip location was confirmed by visualising LA spread lifting the erector spinae muscle off the bony shadow of the transverse process. Once confirmed, $20 \mathrm{ml}$ of $0.25 \%$ bupivacaine was administered in group II under vision after confirming negative aspiration of blood, and $20 \mathrm{ml}$ normal saline was administered in group I. LA distribution was observed in both cranial and caudal directions.

\section{Enrollment}

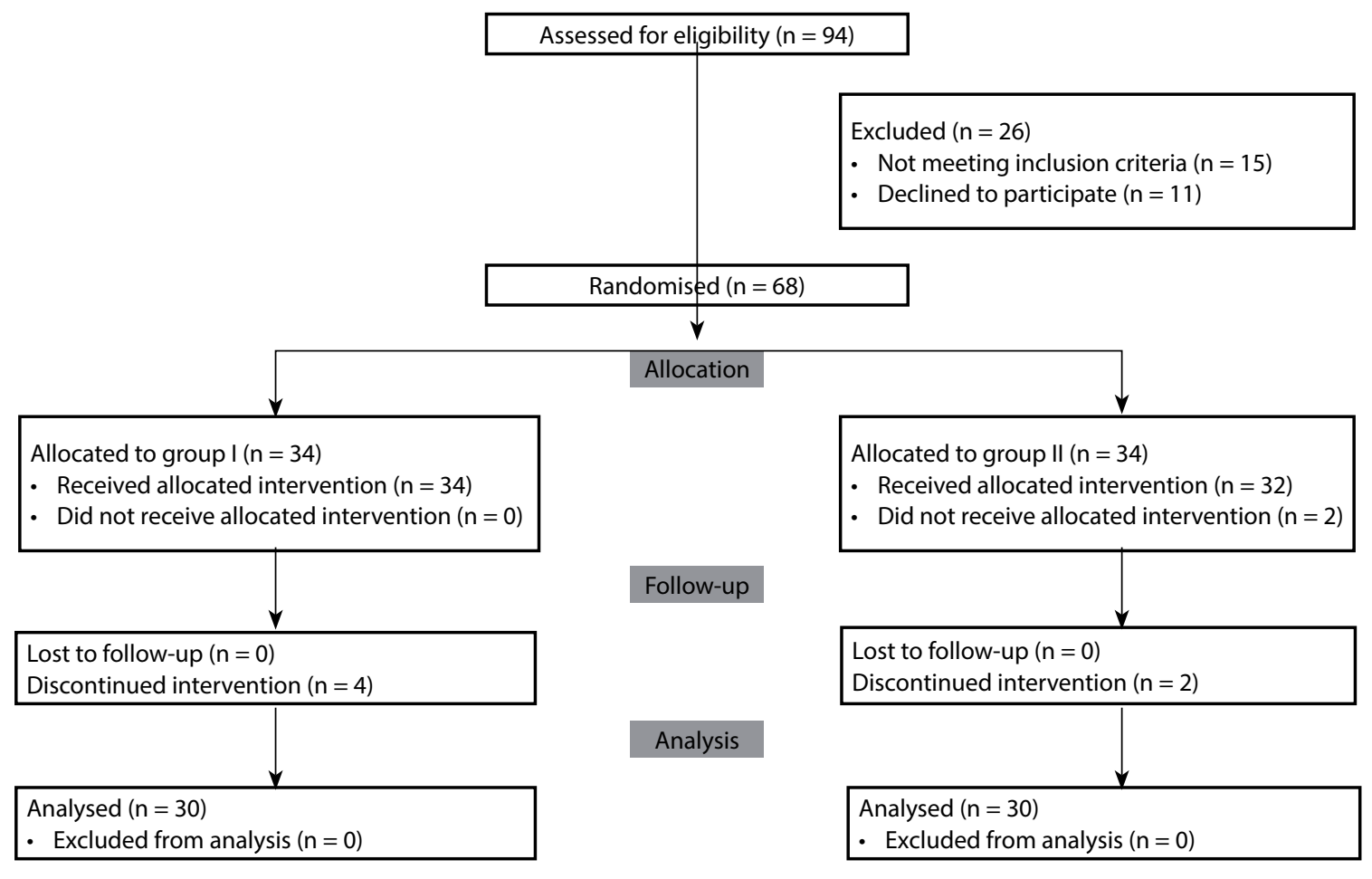

Figure 1. CONSORT flow diagram of participants through each stage of the randomised trial 


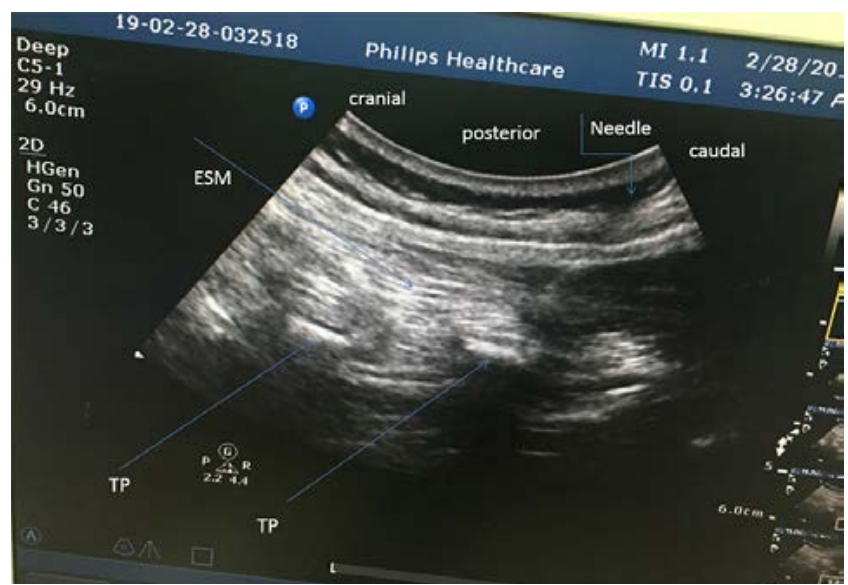

Figure 2. Sonoanatomy at the level of third and fourth lumbar vertebrae shadow of needle advanced toward the transverse process of the third vertebra.

TP - Transverse process; ESM - Erector spinae muscle

This was repeated on the other side after turning the patient to the other side as shown in Figures 2 and 3.

Twenty minutes later, loss of cold sensation was evident between (T10-T12) and (L5-S1) vertebral level of the posterior dermatomes and dermatomes of the anterior roots of the spinal nerves (lumbar plexus, upper leg) on both sides (without haemodynamic changes). Failure of block occurred if the loss of sensation was not attained within 30 minutes.

\section{Anaesthetic management}

Anaesthesia was induced 30 minutes after finishing the USguided ESPB and a crystalloid intravenous infusion of 6 to 8 $\mathrm{ml} / \mathrm{kg} / \mathrm{h}$ was started. Previous monitors were applied plus temperature probe, capnography, and electrodes for monitoring the bispectral index (BISTM, model A-2000s; Aspect Medical Systems, Norwood, MA, USA). Induction was performed using fentanyl $1 \mu \mathrm{g} / \mathrm{kg}$, atracurium $0.5 \mathrm{mg} / \mathrm{kg}$ and propofol $2 \mathrm{mg} /$ $\mathrm{kg}$. Intubation was done by an armoured endotracheal tube of appropriate size. Anaesthesia was maintained with isoflurane $1.5 \%$, atracurium $0.1 \mathrm{mg} / \mathrm{kg}$ as required, and ventilator settings were adjusted to keep $\mathrm{EtCO}_{2}$ between 35 and $40 \mathrm{mmHg}$. The patient was turned to the prone position carefully with careful padding of pressure points and eyes. Intraoperative analgesia was provided by supplementary doses of intravenous fentanyl $(1 \mu \mathrm{g} / \mathrm{kg})$ when heart rate or mean blood pressure increased more than $20 \%$ above the baseline. At the end of the surgery, isoflurane was discontinued, and muscle relaxant was reversed using slow intravenous injection of $0.05 \mathrm{mg} / \mathrm{kg}$ neostigmine and $0.01 \mathrm{mg} / \mathrm{kg}$ atropine sulphate. Careful tracheal extubation was performed at the OR after fulfilling the criteria for extubation (full consciousness, haemodynamic stability with compensated lost blood volume and adequate reversal of neuromuscular blockade).

After extubation, all patients were transferred immediately to the PACU. Patients were discharged from the PACU according to modified Aldrete score (if the score is $\geq 9$, the patient can be discharged). ${ }^{7}$ Intravenous paracetamol $1 \mathrm{gm} / 6$ hours and ketorolac $30 \mathrm{mg}$ loading dose then $15 \mathrm{mg} / 8$ hours regularly.

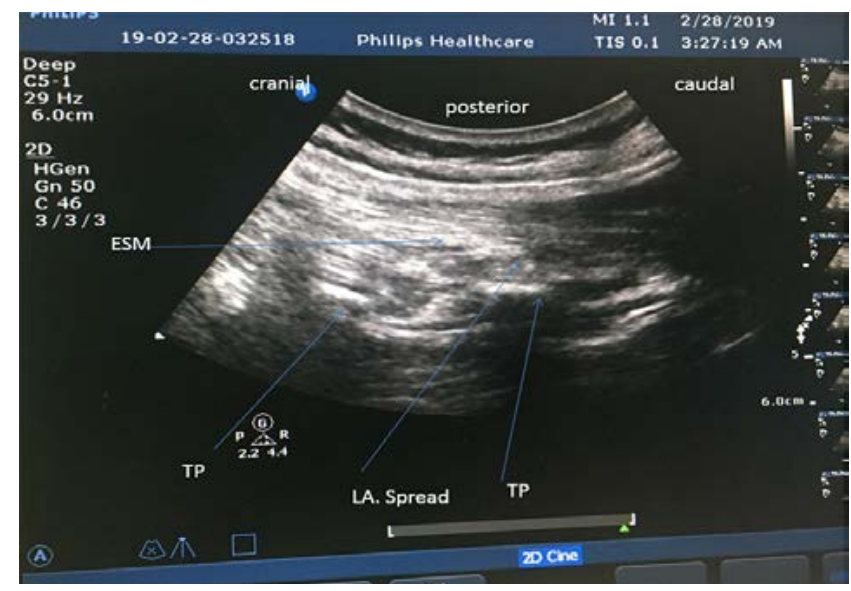

Figure 3. Ultrasound image with the spread of local anaesthetic injection between the transverse process of third lumbar vertebra and fascia of erector spinae muscle TP - Transverse process; ESM - Erector spinae muscle; LA - Local anaesthetic

Morphine $0.1 \mathrm{mg} / \mathrm{kg}$ IV was given as postoperative rescue analgesia if VAS was $>30$.

Our primary outcome was to measure total morphine consumption during the first 24 hours postoperative. Secondary measurements were time till the first request of rescue analgesia which indicated the duration of analgesia and total intraoperative fentanyl consumption. Baseline HR and MBP were recorded before performing ESPB (T1) 5, 10, 15 min after the block (T2T4) and immediately after intubation, then every 15 min until the end of surgery (named T5-T17).

Static VAS (at rest) was assessed immediately after PACU arrival and then after 2, 4, 6, 8, 12, 18 and 24 hours postoperative, while dynamic VAS (during ambulation) was evaluated first 6 hours postoperatively, when patient began to ambulate; length of PACU stay, complications (nausea, vomiting, hypotension, bradycardia, and somnolence) and LA toxicity (e.g., central nervous toxicity in the form of dizziness, tinnitus, numbness of tongue, metallic taste, visual disturbance, and dysarthria) were recorded. The observers who collected data and were responsible for postoperative follow-up were blinded to the study groups.

\section{Statistical analysis}

Based on the results of a previous study which measured total postoperative opioid consumption, ${ }^{8}$ sample size calculation suggested a minimum of 21 patients in each group to detect a $40 \%$ difference in total morphine consumption at 24 hours postoperative (our primary outcome) at a error of 0.05 , standard deviation of 6.92 and power of the study of $85 \%$. Thus, in our study, 30 cases were enrolled in each group to compensate for possible dropouts.

The collected data were analysed using SPSS (SPSS Inc., Chicago, IL, USA) version 25. Normality of data was checked by Shapiro Wilks test and all our data were normally distributed. Quantitative data were presented as mean \pm SD and compared by unpaired T-test. Qualitative data were presented as number and percentage (\%) and compared by the chi-square test. The level of significance was adopted at $p$-value $<0.05$. 
Table I. Demographic data, ASA classification, and duration of surgery in the two groups.

\begin{tabular}{lccc}
\hline Variable & Group I & Group II & P value \\
\hline Age (years) & $42.8 \pm 10.7$ & $43.9 \pm 9.8$ & 0.379 \\
Sex & & & \\
Male & 16 & 17 & 0.727 \\
Female & 14 & 13 & \\
ASA classification & 17 & 15 & 0.730 \\
I & 13 & 15 & \\
II & $82.7 \pm 10.7$ & $86.8 \pm 6.8$ & 0.118 \\
Weight (kg) & $179.4 \pm 16.8$ & $175.5 \pm 13.6$ & 0.199 \\
\hline $\begin{array}{l}\text { Duration of } \\
\text { surgery (min) }\end{array}$ & & & \\
\hline
\end{tabular}

Data presented as mean \pm SD or patient's number. SD - Standard deviation; ASA - American Society of Anesthesiologists.

Table II. Intra- and postoperative opioid consumption, time to first request of analgesia, length of PACU stay, and complications in the two groups.

\begin{tabular}{lccc}
\hline Variable & Group I & Group II & P value \\
\hline $\begin{array}{l}\text { Intraoperative } \\
\text { fentanyl } \\
\text { consumption ( } \mu \text { g) }\end{array}$ & $298.2 \pm 16.3$ & $75.5 \pm 5.99$ & $0.001^{*}$ \\
$\begin{array}{l}\text { 24 h postoperative } \\
\text { morphine }\end{array}$ & $29.2 \pm 6.13$ & $24.95 \pm 2.69$ & $0.001^{*}$ \\
$\begin{array}{l}\text { consumption (mg) } \\
\text { Time of first }\end{array}$ & & & \\
$\begin{array}{l}\text { analgesic request } \\
\text { (min) }\end{array}$ & $178.33 \pm 45.26$ & $461.33 \pm 58.82$ & $0.001^{*}$ \\
PACU stay (min) & $103.2 \pm 10.1$ & $70.4 \pm 12.3$ & $<0.001^{*}$ \\
$\begin{array}{l}\text { Nausea } \\
\text { Vomiting }\end{array}$ & $5(16.7 \%)$ & $2(6.7 \%)$ & 0.424 \\
Somnolence & $3(10 \%)$ & 0 & 0.237 \\
\hline
\end{tabular}

Data presented as mean \pm SD. SD - Standard deviation; \% - percentage; PACU - Post-anaesthesia care unit. ${ }^{*}$ denotes statistically significant difference at $P$ value $<0.05$

\section{Results}

In our study, 94 patients were assessed for eligibility; 15 patients did not meet the inclusion criteria and 11 patients refused to participate in the study. Sixty-eight patients were randomised into 2 equal groups (34 patients in each one). Two patients in group II did not receive the intervention because they were uncooperative. Four patients in group I and two patients in group II were not followed up because they refused to continue the intervention. Thirty patients in each group were analysed as shown in Figure 1.

Patients enrolled in both groups were comparable for demographic data (age, weight, and gender), ASA classification and duration of surgery (Table I).

It was found that time until the first request of rescue analgesia was significantly prolonged in group II as compared to group I. Intraoperative fentanyl and postoperative 24 hour morphine consumption was significantly increased in group I as compared to group II. Length of PACU stay was significantly shorter in group II than in group I. The results are summarised in Table II.
The results showed that pain scores began to increase in group II at 12 and 8 hours postoperative (static and dynamic VAS respectively), but they were lower than in group I. With regard to static VAS, there was significant difference between both groups on arrival to PACU 2, 4, 6 and 8 hours postoperatively ( $p<0.001$ ), while dynamic VAS was significantly lower in group II than group I at 6,8 and 12 hours postoperative $(p<0.001)$ as shown in Figures 4 and 5 .

The obtained results showed significant increase in HR and MBP in group I rather than in group II at T6, T8, T9, T10, T11, T12, T13, T15 and T16, as shown in Figures 6 and 7.

Incidence of complications (nausea, vomiting, and somnolence) was lower in group II than in group I. Somnolence was significantly lower in group II. No other complications were observed as summarised in Table II.

\section{Discussion}

The purpose of the study is to use ESPB to provide effective analgesia after PLIF surgeries. Intraoperative and postoperative opioid consumption was reduced in patients who received ESPB and VAS score was better with prolonged time to request of rescue analgesia. Shorter PACU stay and lower complications were observed in patients who received ESPB.

In our study, ESPB was performed by injection of LA in the fascial plane deep to the erector spinae muscle, from where it diffuses to the dorsal and ventral rami of the spinal nerves, achieving an extensive multi-dermatomal sensory block at posterior,

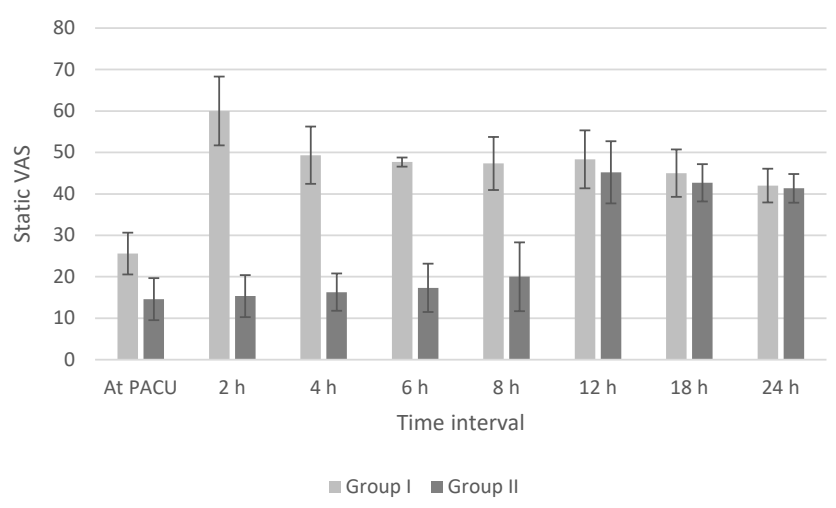

Figure 4. Static visual analog scale (VAS) changes in the two groups. Data presented as mean \pm SD.

PACU - Post-anaesthesia care unit

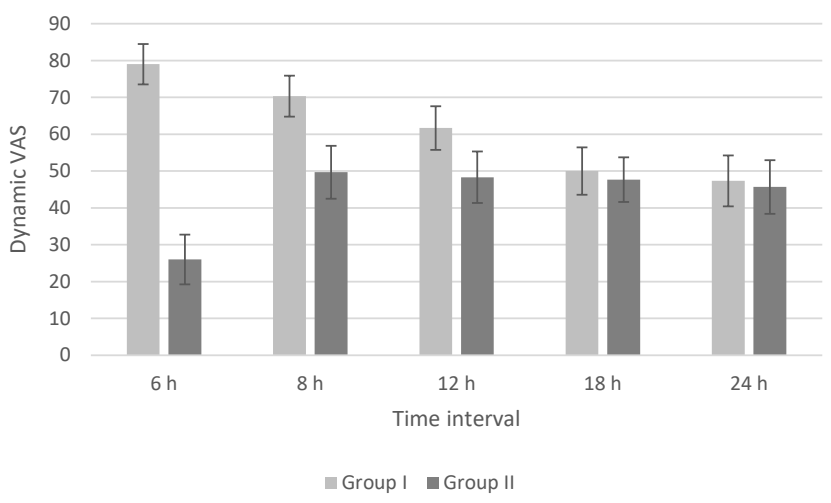

Figure 5. Dynamic visual analog scale (VAS) changes in the two groups. Data presented as mean \pm SD. 


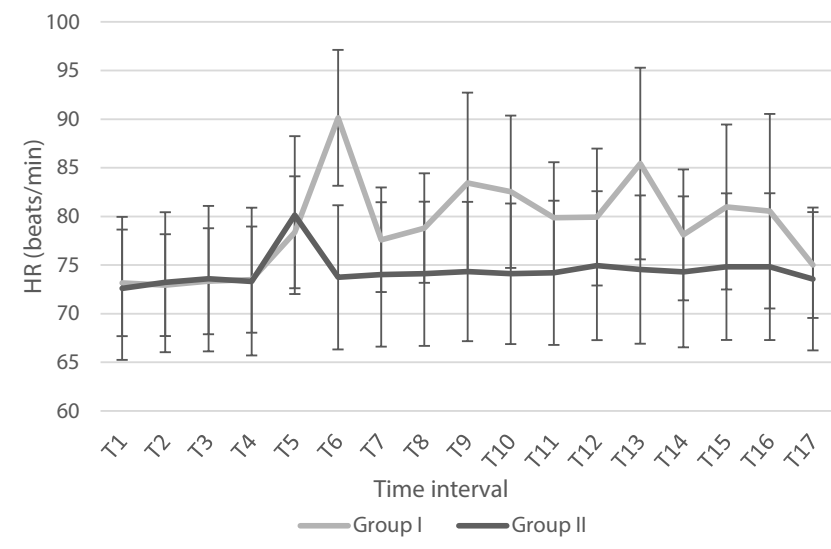

Figure 6. Heart rate (beat/min) changes in the two groups. Data presented as mean \pm SD.

HR - heart rate; T1 - baseline; T2-4 - 5,10,15 min after block; T5-7 - immediately after intubation then every $15 \mathrm{~min}$ till end of the surgery

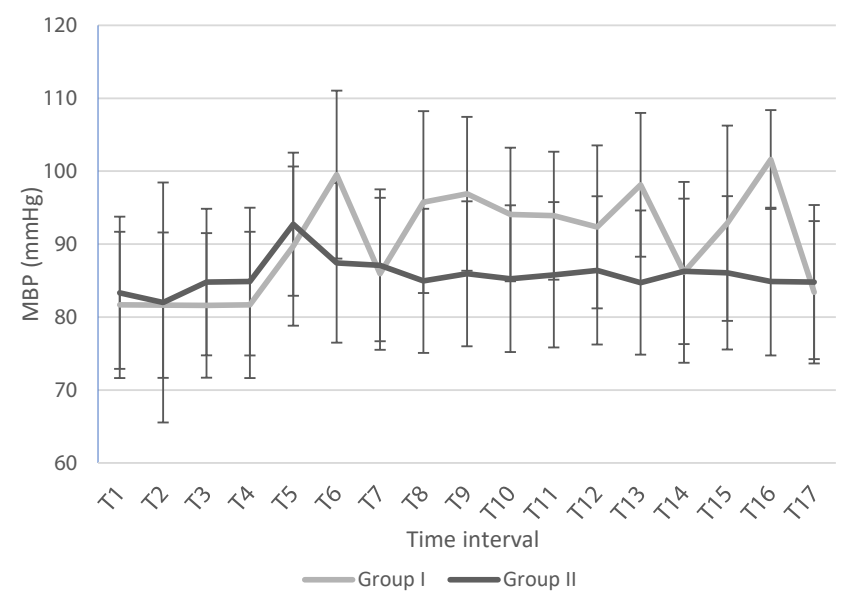

Figure 7. Mean arterial blood pressure $(\mathrm{mmHg})$ changes in the two groups. Data presented as mean $\pm S D$.

MAP - mean arterial pressure; MBP - mean blood pressure; T1 - baseline;

T2-4 - 5,10,15 min after block; T5-7 - immediately after intubation then every 15 min till the end of surgery

lateral, and anterior aspect. ${ }^{9}$ Therefore ESPB can be used in pain management of lumbar spondylolisthesis. ${ }^{10,11}$

These results are in agreement with other studies that have shown that ESPB performed safely and effectively for perioperative analgesia in lumbosacral spine surgery. The choice was the T10 or T12 transverse process level in six cases of lumbosacral spine surgery for their bilateral ESP blocks, targeting sensory block between L2-S1. ${ }^{1}$ Also, adequate and effective ESP blocks at the level of the $\mathrm{L} 2$ transverse process were done by Takahashi et al. ${ }^{10}$ Their target was to block an area extending from T12 to L 5 but they used $20 \mathrm{ml}$ of $0.1875 \%$ ropivacaine.

Chaudhary NK et al. ${ }^{11}$ reported that bilateral ESP blocks were a safe and effective technique for postoperative pain management after spine surgery. Prolonged analgesia was maintained by inserting a catheter in the interfascial plane deep to the erector spinae muscle in a case series study. They chose the level of T10 transverse process for blocks and $20 \mathrm{ml} 0.25 \%$ bupivacaine through both the catheters, targeting a sensory blockade between T7-T 8 and L2-L3 vertebral level in the anterior, lateral, and posterior part on both sides. In addition, Chin KJ et al. ${ }^{12}$ suggested the use of adjuncts and catheter insertion for intermittent boluses or continuous infusions of local anaesthetic to prolong analgesia of ESPB.

The findings of our study are consistent with some studies ${ }^{13-15}$ with different types of surgeries that demonstrated that duration of the analgesia after single shot ESPB was around 10 hours or more.

Based on previous trials, ${ }^{10,11}$ we do ESPB at the level of $L 3$ by 20 $\mathrm{ml} 0.25 \%$ bupivacaine targeting to block the area between $\mathrm{T} 12$ and S1.

The data obtained are broadly consistent with Melvin JP et al. ${ }^{1}$ who stated that pre-incision ESP blocks performed at the T10-T12 level contributed to effective perioperative opioidsparing analgesia and enhanced recovery in a preliminary series of six patients undergoing lumbosacral spine surgery.

Ueshima $\mathrm{H}$ and Otake $\mathrm{H}$ studied two cases undergoing thoracic vertebra surgery and they found that there was no need for additional analgesic for perioperative pain relief. Thus, ESP blocks were able to block the dorsal and ventral rami of the thoracic spinal nerves and are useful for good perioperative pain control of thoracic vertebra surgery. ${ }^{16}$

Our results were in agreement with Takahashi $\mathrm{H}$ et al. ${ }^{17}$ who stated that ESPB is an easy and safe procedure used in failed back surgery syndrome with associated low back pain.

Compared with other regional techniques, ESPB is a safe and simple technique. The epidural analgesia involves the midline plane which is at the surgical site and is not preferred by many surgeons. ${ }^{2}$ Lumbar paravertebral block has the potential of devastating complications, such as intrathecal or intramedullary injection, as the nerve roots are surrounded by dura, and root level blocks are done just outside the dura. ${ }^{18}$

\section{Study limitations}

The limitation of the study results is the relatively short duration of the study. The postoperative follow-up should be for a longer time with the possibility of continuous US-guided ESPB by catheter insertion into the plane which may increase sensory block duration. Further studies are recommended to compare with other drugs (e.g., ropivacaine, levobupivacaine), other additives (e.g., dexamethasone, opioids) and other techniques (e.g., paravertebral block).

\section{Conclusion}

In conclusion, it has been demonstrated that effective acute postoperative pain control in patients with L3-L5 spondylolisthesis undergoing PLIF could be managed with bilateral single shot US-guided ESPB at the level of L3. Also, ESPB shortened PACU stay without adverse effects.

\section{Acknowledgments}

This research did not receive any financial support. 


\section{Conflict of interest}

\section{No conflict of interest.}

\section{References}

1. Melvin JP, Schrot RJ, Chu GM, Chin KJ. Low thoracic erector spinae plane block for perioperative analgesia in lumbosacral spine surgery: a case series. Can J Anaesth. 2018;65(9):1057-65. Available from: https://doi.org/10.1007/ s12630-018-1145-8

2. Sharma S, Balireddy RK, Vorenkamp KE, Durieux ME. Beyond opioid patientcontrolled analgesia: a systematic review of analgesia after major spine surgery. Reg Anesth Pain Medicine. 2012;37(1):79-98. Available from: https://doi. org/10.1097/AAP.0b013e3182340869

3. Adhikary SD, Bernard S, Lopez H, Chin KJ. Erector spinae plane block versus retrolaminar block: a magnetic resonance imaging and anatomical study. Reg Anesth Pain Medicine. 2018;43(7):756-62. Available from: https://doi. org/10.1097/AAP.0000000000000798

4. Nicholson A, Lowe M, Parker J, Lewis S, Alderson P, Smith A. Systematic review and meta-analysis of enhanced recovery programmes in surgical patients. $\mathrm{Br} J$ Surg. 2014;101(3):172-88. Available from: https://doi.org/10.1002/bjs.9394

5. Devin CJ, McGirt MJ. The best evidence in multimodal pain management in spine surgery and means of assessing postoperative pain and functional outcomes. J Clin Neurosci. 2015;22(6):930-8. Available from: http://dx.doi. org/10.1016/j.jocn.2015.01.003

6. Forero M, Adhikary SD, Lopez H, Tsui C, Chin KJ: The erector spinae plane block: a novel analgesic technique in thoracic neuropathic pain. Reg Anesth Pain Med. 2016;41(5):621-7. Available from: http://dx.doi.org/10.1097/ AAP.0000000000000451

7. Aldrete JA. The post-anesthesia recovery score revisited. J Clin Anesth. 1995;7(1):89-91.

8. Gürkan Y, Aksu C, Kuş A, Yörükoğlu UH, Kılıç CT. Ultrasound-guided erector spinae plane block reduces postoperative opioid consumption following breast surgery: a randomized controlled study. J Clin Anesth. 2018;50:65-8. Available from: https://doi.org/10.1016/j.jclinane.2018.06.033
9. López MB, Cadórniga ÁG, González JML, Suárez ED, Carballo CL, Sobrino FP. Erector spinae block. A narrative review. Cent Eur J Clin Res. 2018;1(1):28-39. Available from: https://doi.org/10.2478/cejcr-2018-0005

10. Takahashi H, Suzuki T. Erector spinae plane block for low back pain in failed back surgery syndrome: a case report. JA Clinical Reports. 2018;4(1):60. Available from: https://doi.org/10.1186/s40981-018-0198-6

11. Chaudhary NK, Singh S. Continuous ultrasound-guided erector spinae plane block for post-operative pain management in lumbar spine surgery: A case series. Indian J Anaesth. 2018;62(8):638-9. Available from: https://doi.org/ 10.4103/ija.IJA_160_18

12. Chin K, Adhikary S, Sarwani N, Forero M. The analgesic efficacy of preoperative bilateral erector spinae plane (ESP) blocks in patients having ventral hernia repair. Anaesthesia. 2017;72(4):452-60. Available from: https:// doi:10.1111/ anae.13814

13. Tulgar S, Selvi O, Senturk O, Ermis MN, Cubuk R, Ozer Z. Clinical experiences of ultrasound-guided lumbar erector spinae plane block for hip joint and proximal femur surgeries. J Clin Anesth. 2018; 47:5-6. Available from: https://doi. org/10.1016/j.jclinane.2018.02.014

14. Tulgar S, Selvi O, Kapakli MS. Erector spinae plane block for different laparoscopic abdominal surgeries: case series. Case Rep Anesthesiol. 2018 Feb 18;2018:3947281. Available from: https://doi.org/10.1155/2018/3947281

15. Krishna SN, Chauhan S, Bhoi D, et al. Bilateral Erector Spinae Plane block for Acute Post-Surgical pain in Adult Cardiac Surgical patients: a randomized controlled trial. J Cardioth Vasc Anesth. 2019;33(2):368-75. Available from: https://doi.org/10.1053/j.jvca.2018.05.050

16. Ueshima $H$, Otake $H$. Clinical experiences of ultrasound-guided erector spinae plane block for thoracic vertebra surgery. J Clin Anesth. 2017;38:137. Available from: https://doi.org/ 10.1016/j.jclinane.2016.12.028

17. Takahashi H, Suzuki T. Erector spinae plane block for low back pain in failed back surgery syndrome: a case report. JA Clinical Reports. 2018;4(1):60-3. Available from: https://doi.org/10.1186/s40981-018-0198-6

18. Boezaart AP, Lucas SD, Elliott CE. Paravertebral block: cervical, thoracic, lumbar and sacral. Curr Opin Anaesthesiol. 2009;22(5):637-43. Available from: https:// doi.org/10.1097/ACO.0b013e32832f3277 